\title{
On D-Preopen Sets in D-Matric Spaces
}

\author{
Hussain Wahish \\ Department of Mathematics,Faculty \\ of Education, University of Saba, \\ Region, Mareb, Yemen
}

\author{
Amin Saif \\ Department of Mathematics, Faculty of \\ Applied Sciences, Taiz University, \\ Taiz, Yemen
}

\begin{abstract}
The purpose of this paper is to introduce and investigate weak form of D-open sets in D-metric spaces, namely D-preopen sets. The relationships among this form with the other known sets are introduced. We give the notions of the interior operator, the closure operator and frontier operator via D-preopen sets.
\end{abstract}

AMS classification: Primary 54A05, 54E35.

\section{Keywords}

Open set, Metric spaces.

\section{INTRODUCTION}

Metric spaces is one of the most important spaces in mathematics there are various type of generalization of metric spaces, [5]. The axiomatic approach to the metric spaces is given by a french mathematician M. Frechet in year 1812, [7]. In 1984, Dhage, [3], introduced a new notion of a new structure of D-metric space which is a natural generalization of the notion of ordinary metric space to higher dimensional metric spaces, [4]. In 2000, Dhage, [2], introduced some results in D-metric spaces are obtained and the notion of open and closed balls. In 2013, [6], exhibited methods of generating D-metrics from certain types of real valued partial functions on the three dimensional Euclidean space. In 2017, Ali Fora, Massadeh and Bataineh, [1], introdused and a new topological structure of D-closed set.

This paper is organized as follows. Section 2 is devoted to some preliminaries. Section 3 introduces the concept of D-preopen sets by utilizing the D-open balls. Furthermore, the relationship with the other known sets will be studied. In Section 4 we introduce the concepts of the interior operator, the closure operator and frontier operator via D-preopen sets.

\section{PRELIMINARIES}

Definition 2.1. 77. Let $X$ be any nonempty set. A function $d: X \times X \rightarrow[0, \infty)$ is called a metric function on $X$ if it satisfies the following three conditions for all $x, y, z \in X$ :

(1) (positive property) $d(x, y) \geq 0$ with equality if and only if $x=y$;

(2) (symmetric property) $d(x, y)=d(x, y)$;

(3) (triangle inequality) $d(x, z) \leq d(x, y)+d(y, z)$.
A pair $(X, d)$, where $d$ is a metric on $X$ is called a metric space. By $O_{\varepsilon}(x)$, we mean the open ball with center $x$ and radius $\varepsilon>0$, that is,

$$
O_{\varepsilon}(x)=\{y \in X: d(x, y)<\varepsilon\} .
$$

By $C_{\varepsilon}(x)$, we mean the closed ball with center $x$ and radius $\varepsilon>0$, that is,

$$
C_{\varepsilon}(x)=\{y \in X: d(x, y) \leq \varepsilon\} .
$$

For metric space $(X, d)$ and $G \subseteq X$, the set $G$ said to be open set if for any point $x \in G$, there exists $\varepsilon>0$ such that $O_{\varepsilon}(x) \subseteq G$. The set $G$ is called closed set in metric space $(X, d)$ if $X-G$ is an open set in metric space $(X, D)$. For the set of real numbers $R$, we mean by the usual metric space $(R, d)$,

$$
d(x, y)=|x-y| \text { for all } x, y \in R
$$

For metric space $(X, d)$ and $G \subseteq X$, the interior operator of $G$ is denoted by $\operatorname{Int}(G)$ and the clouser operator of $G$ is denoted by $C l(G)$.

DEFINITION 2.2. [4]. A nonempty set $X$, together with a function $D: X \times X \times X \rightarrow[0, \infty)$ is called a D-metric space, denoted by $(X, D)$ if $D$ satisfies the following $x, y, z, u \in X$ :

(1) $D(x, y, z)=0 \rightarrow x=y=z$ (coincidence);

(2) $D(x, y, z)=D(p(x, y, z))$, where $p$ is a permutation of $x, y, z$ (symmetry);

(3) $D(x, y, z) \leq D(x, y, a)+D(x, a, z)+D(a, y, z)$ for all $x, y, z, u \in X$ (tetrahedral inequality).

By $O_{\varepsilon}^{D}(x)$, we mean the D-open ball with center $x$ and radius $\varepsilon>$ 0 , that is,

$$
O_{\varepsilon}^{D}(x)=\{y \in X: d(x, y, y)<\varepsilon\} .
$$

By $C_{\varepsilon}^{D}(x)$, we mean the D-closed ball with center $x$ and radius $\varepsilon>0$, that is,

$$
C_{\varepsilon}^{D}(x)=\{y \in X: d(x, y, y) \leq \varepsilon\} .
$$

The set $G \subseteq X$ is called D-open set in D-metric space $(X, D)$ if for every $x \in G$, there is $\varepsilon>0$ such that $O_{\varepsilon}^{D}(x) \subseteq G$. The set $G$ is called D-closed set in D-metric space $(X, D)$ if $X-G$ is D-open set in D-metric space $(X, D)$. For D-metric space $(X, D)$ and $G \subseteq X$, the interior set of $G$ is denoted by $\operatorname{Int}_{D}(G)$ and the clouser set of $G$ is denoted by $C l_{D}(G)$.

THEOREM 2.3. [2]. Let $(R, D)$ be D-metric space where

$$
D(x, y, z)=\max \{d(x, y), d(y, z), d(z, x)\}
$$


and $(R, d)$ is usual metric space. Then for a fixed $x \in R$, the Dopen balls $O_{\varepsilon}^{D}(x)$ and $O_{\varepsilon}^{D}(x)$ are the sets in given by: $O_{\varepsilon}^{D}(x)=$ $(x-\varepsilon, x+\varepsilon)$.

THEOREM 2.4. [2]. Let $(R, D)$ be D-metric space, where

$$
D(x, y, z)=d(x, y)+d(y, z)+d(z, x)
$$

and $(R, d)$ is usual metric space. Then for a fixed $x \in R$, the Dopen balls $O_{\varepsilon}^{D}(x)$ and $O_{\varepsilon}^{D}(x)$ are the sets in given by: $O_{\varepsilon}^{D}(x)=$ $(x-\varepsilon / 2, x+\varepsilon / 2)$.

Theorem 2.5. [2]. Every D-open $O_{\varepsilon}^{D}(\mathrm{x}), x \in X, \varepsilon>0$ is a D-open set in $X$ (i.e., it contains a ball of each of its points).

THEOREM 2.6. [1]. Every a finite set in a D-metric space $(X, D)$ must be $\mathrm{D}$-closed set.

THEOREM 2.7. [2]. Every ball $C_{\varepsilon}^{D}(x)$ in a D-metric space $(X, D)$ is $\mathrm{D}$-closed set.

THEOREM 2.8. [2]. Arbitrary union and finite intersection of D-open balls $O_{\varepsilon}^{D}(x), x \in X$ is D-open set.

THEOREM 2.9. [1]. Let $D: X \times X \times X \times X \rightarrow[0, \infty)$ be a D-metric on $X$ having a finite range. Then every subset $A$ of $X$ is D-closed set.

\section{D-PREOPEN SETS}

Definition 3.1. Let $(X, D)$ be a D-metric space. A subset $G \subseteq X$ is called a D-preopen set in D-metric space $(X, D)$ if for every $x \in G$, there is $\delta>0$ such that for every $y \in O_{\delta}^{D}(x)$, $O_{\varepsilon}^{D}(y) \cap G \neq \emptyset$ for every $\varepsilon>0$.A subset $G \subseteq X$ is called a D-preclosed set in D-metric space $(X, D)$ if $X-\bar{G}$ is a D-preopen set in D-metric space $(X, D)$.

The set of all D-preopen sets in $X$ denoted by $D_{p} O(X, D)$ and the set of all D-preclosed sets in $X$ denoted by $D_{p} C(X, D)$.

EXAMPLE 3.2. Let $(R, D)$ be D-metric space given by

$$
D(x, y, z)=\max \{d(x, y), d(y, z), d(z, x)\},
$$

where $(R, d)$ is usual metric space on the set of real number $R$. An open interval $G=(0,2)$ is D-preopen set in $(R, D)$. For every $x \in G$, take $\delta=\min \{|x|,|2-x|\}>0$. If $y \in O_{\delta}^{D}(x)$, then $O_{\varepsilon}^{D}(y) \cap G \neq \emptyset$ for every $\varepsilon>0$.

EXAMPLE 3.3. In Example 3.2, a closed interval $G=[-1,1]$ is not D-preopen set, since at $\mathrm{x}=1$, take $y=(2+\delta) / 2 \in O_{\delta}^{D}(1)$ and $\varepsilon=\delta / 2>0$. Note that $O_{\delta / 2}^{D}((2+\delta) / 2) \cap G=\emptyset$. That is, $G=[-1,1]$ is not D-preopen set in $(R, D)$.

TheOREM 3.4. Every D-open set is a D-preopen set.

Proof. Let $G$ be any D-open set in D-metric space $(X, D)$. Let $x \in G$ be arbitrary point. Then there is $\delta>0$ such that $O_{\delta}^{D}(x) \subseteq$ $G$. For every $y \in O_{\delta}^{D}(x), y \in O_{\varepsilon}^{D}(y)$ and $y \in G$ for every $\varepsilon>0$. That is, $O_{\varepsilon}^{D}(y) \cap G \neq \emptyset$ for every $\varepsilon>0$. Hence $G$ is D-preopen set.

The converse of above theorem need not be true.

EXAMPLE 3.5. In Example 3.2), the set of rational numbers $Q$ is a $\mathrm{D}$-preopen set but not $\mathrm{D}$-open set in $(R, D)$.

Note that the intersection of two D-preopen sets no need to be D-preopen set. In Example 3.2, the set of rational numbers $Q$ is a $\mathrm{D}$-preopen set but not D-open set in $(R, D)$ and the set $I R \cup\{q\}$ is a D-preopen set in $(R, D)$, where $I R$ is the set of irrational numbers and $q$ is any rational number, but $Q \cap(I R \cup\{q\})=\{q\}$ is not D-preopen set.

The following theorem shows that the intersection of a D-open set and a D-preopen set is a D-preopen set.

ThEOREM 3.6. The intersection of a D-open set and a Dpreopen set is a D-preopen set.

Proof. Let $A$ be D-open set and $B$ be D-preopen set in Dmetric space in $(X, D)$. Let $x \in A \cap B$ be arbitrary point. Then $x \in A$ and $x \in B$. Then there are $\delta_{1}>0$ and $\delta_{2}>0$ such that $O_{\delta_{1}}^{D}(x) \subseteq A$ and for every $y \in O_{\delta_{2}}^{D}(x), O_{\varepsilon}^{D}(y) \cap B \neq \emptyset$ for every $\varepsilon>0$. Take $\delta=\min \left\{\delta_{1}, \delta_{2}\right\}>0$. Then $O_{\delta}^{D}(x) \subseteq A$ and for every $y \in O_{\delta}^{D}(x), O_{\varepsilon}^{D}(y) \cap B \neq \emptyset$ for every $\varepsilon>0$. Now for every $y \in O_{\delta}^{D}(x)$ and since $A$ is D-open set, then there is $\varepsilon_{y}>0$ such that $O_{\varepsilon_{y}}^{D}(y) \subseteq A$ and $O_{\min \left\{\varepsilon_{y}, \varepsilon\right\}}^{D}(y) \cap B \neq \emptyset$. Since $O_{\min \left\{\varepsilon_{y}, \varepsilon\right\}}^{D}(y) \cap B \subseteq O_{\varepsilon}^{D}(y) \cap A \cap B$, then $O_{\varepsilon}^{D}(y) \cap(A \cap B) \neq \emptyset$ for every $\varepsilon>0$. That is $A \cap B$ is D-preopen set.

THEOREM 3.7. The union of any family of D-preopen sets is D-preopen set.

Proof. Let $G_{\lambda}$ be a D-preopen subset of D-metric space $(X, D)$ for all $\lambda \in \Delta$. Let $x \in \bigcup_{\lambda \in \Delta} G_{\lambda}$ be an arbitrary point. Then there is at least $\lambda_{0} \in \Delta$ such that $x \in G_{\lambda_{0}}$. Since $G_{\lambda_{0}}$ is a D-preopen then for every $x \in G_{\lambda_{0}}$, there is $\delta>0$ such that for every $y \in O_{\delta}^{D}(x), O_{\varepsilon}^{D}(y) \cap G_{\lambda_{0}} \neq \emptyset$ for every $\varepsilon>0$. Since $G_{\lambda_{0}} \subseteq \bigcup_{\lambda \in \Delta} G_{\lambda}$, then for every $\mathrm{x} \in \bigcup_{\lambda \in \Delta} G_{\lambda}$, there is $\delta>0$ such that for every $y \in O_{\delta}^{D}(x), O_{\varepsilon}^{D}(y) \cap \bigcup_{\lambda \in \Delta} G_{\lambda} \neq \emptyset$ for every $\varepsilon>0$. That is $\bigcup_{\lambda \in \Delta} G_{\lambda}$ is D-preopen set.

\section{D-PREOPEN OPERATORS}

In this section, we define the interior operator, the closure operator and frontier operator via D-preopen sets.

Definition 4.1. Let $(X, D)$ be a D-metric space and $G \subseteq X$. The $D_{P}$-closure operator of $G$ is denoted by $C l_{P}^{D}(G)$ and defined by

$C l_{P}^{D}(G)=\cap\{H \subseteq X: G \subseteq H$ and $H$ is D-preclosed set $\}$.

The $D_{P}$-interior functor of $G$ is denoted by $\operatorname{Int}_{P}^{D}(G)$ and defined by

$$
\begin{aligned}
& \operatorname{Int} t_{P}^{D}(G)=\cup\{H \subseteq X: H \subseteq G \text { and } H \text { is D-preopen set }\} . \\
& \text { REMARK 4.2. }
\end{aligned}
$$

(1) From Theorem 3.7), $C l_{P}^{D}(G)$ is a D-preclosed set and $\operatorname{Int}_{P}^{D}(G)$ is D-preopen set in D-metric space $(X, D)$.

(2) For a D-metric space $(X, D)$ and $G \subseteq X$, it is clear from the definition of $C l_{P}^{D}(G)$ and $\operatorname{Int}_{P}^{D}(G)$ that $G \subseteq C l_{P}^{D}(G)$ and $\operatorname{Int} t_{P}^{D}(G) \subseteq G$.

THEOREM 4.3. For a D-metric space $(X, D)$ and $G \subseteq X$, $C l_{P}^{D}(G)=G$ if and only if $G$ is a D-preclosed set.

Proof. Let $C l_{P}^{D}(G)=G$. Then from definition of $C l_{P}^{D}(G)$ and Theorem (3.7), $C l_{P}^{D}(G)$ is a D-preclosed set and $G$ is a Dpreclosed set. Conversely, we have $G \subseteq C l_{P}^{D}(G)$ by Remark 4.2. Since $G$ is a D-preclosed set, then it is clear from the definition of $C l_{P}^{D}(G), C l_{P}^{D}(G) \subseteq G$. Hence $G=C l_{P}^{D}(G)$.

Theorem 4.4. For a D-metric space $(X, D)$ and $G \subseteq X$, and $\operatorname{Int}_{P}^{D}(G)=G$ if and only if $\mathrm{G}$ is a D-preopen set. 
Proof. Let $G$ be D-preopen set. Then for all $x \in G$, we have $x \in G \subseteq G$. That is, $G \subseteq \operatorname{Int}_{P}^{D}(G)$. Then $G=\operatorname{Int} t_{P}^{D}(G)$ from Remark $\sqrt{4.2}$. The converse is trivial.

THEOREM 4.5. For a D-metric space $(X, D)$ and $G \subseteq X, x \in$ $C l_{P}^{D}(G)$ if and only if for all D-preopen set $M$ containing $x, M \cap$ $G \neq \emptyset$.

Proof. Let $x \in C l_{P}^{D}(G)$ and $M$ be any D-preopen set containing $x$. If $M \cap G=\emptyset$ then $G \subseteq X-M$. Since $X-M$ is a D-preclosed set containing $G$, then $C l_{P}^{D}(G) \subseteq X-M$ and so $x \in C l_{P}^{D}(G) \subseteq X-M$. Hence this is contradiction, because $x \in M$. Therefore $M \cap G \neq \emptyset$.

Conversely, Let $x \notin C l_{P}^{D}(G)$. Then $X-C l_{P}^{D}(G)$ is a D-preopen set containing $x$. Hence by hypothesis, $\left[X-C l_{P}^{D}(G)\right] \cap G \neq \emptyset$. But this is contradiction, because $X-C l_{P}^{D}(G) \subseteq X-G$.

Theorem 4.6. For a D-metric space $(X, D)$ and $G \subseteq X, x \in$ $\operatorname{Int}_{P}^{D}(G)$ if and only if there is D-preopen set $M$ such that $x \in$ $M \subseteq G$.

Proof. Let $x \in \operatorname{Int}_{P}^{D}(G)$ and take $M=\operatorname{Int} t_{P}^{D}(G)$. Then by Theorem 4.5 and definition of $\operatorname{Int}_{P}^{D}(G)$ we get that $M$ is a Dpreopen set and by Remark (4.2), $x \in M \subseteq G$. Conversely, let there is D-preopen set $M$ such that $x \in M \subseteq G$ Then by definition of $\operatorname{Int}_{P}^{D}(G), x \in M \subseteq \operatorname{Int}{ }_{P}^{D}(G)$.

Theorem 4.7. For a D-metric space $(X, D)$ and $G, M \subseteq X$, the following hold:

(1) If $G \subseteq M$ then $C l_{P}^{D}(G) \subseteq C l_{P}^{D}(M)$.

(2) $C l_{P}^{D}(G) \cup C l_{P}^{D}(M) \subseteq C l_{P}^{D}(G \cup M)$.

(3) $C l_{P}^{D}(G \cap M) \subseteq C l_{P}^{D}(G) \cap C l_{P}^{D}(M)$.

(4) $C l_{P}^{D}(G) \subseteq C l_{D}(G)$.

Proof. (1) Let $x \in C l_{P}^{D}(G)$. Then by Theorem 4.5, for all D-preopen set $N$ containing $x, N \cap G \neq \emptyset$. Since $G \subseteq M$ then $N \cap M \neq \emptyset$. Hence $x \in C l_{P}^{D}(M)$. That is, $C l_{P}^{D}(\bar{G}) \subseteq$ $C l_{P}^{D}(M)$.

(2) Since $G \subseteq G \cup M$ and $M \subseteq G \cup M$, then by part(1), $C l_{P}^{D}(G) \subseteq C l_{P}^{D}(G \cup M)$ and $C l_{P}^{D}(M) \subseteq C l_{P}^{D}(G \cup M)$. Hence $C l_{P}^{\bar{D}}(G) \cup C l_{P}^{D}(M) \subseteq C l_{P}^{D}(G \cup M)$.

(3) Since $G \cap M \subseteq G$ and $G \cap M \subseteq M$, then by part(1), $C l_{P}^{D}(G \cap M) \subseteq C l_{P}^{D}(G)$ and $C l_{P}^{D}(G \cap M) \subseteq C l_{P}^{D}(M)$. Hence $C l_{P}^{D}(G \cap M) \subseteq C l_{P}^{D}(G) \cap C l_{P}^{D}(M)$.

(4) It is clear from Theorem 4.5 and from every D-open set is D-preopen set.

In the above theorem $C l_{P}^{D}(G \cup M) \neq C l_{P}^{D}(G) \cup C l_{P}^{D}(M)$ as it is shown in the following example.

EXAMPLE 4.8. Let $(R, D)$ be D-metric space, where

$$
D(x, y, z)=d(x, y)+d(y, z)+d(z, x)
$$

and $(R, d)$ is usual metric space. Let $G=I R$ and $M=Q-[\{q\}]$, where $Q$ is the set of rational numbers, $I R$ is the set of irrational numbers and $q$ is any rational number. Since $G$ and $M$ are Dprecloced sets in $R$. Then $C l_{P}^{D}(G) \cup C l_{P}^{D}(M)=G \cup M=R-$ $\{q\}$. If $R-\{q\}$ is D-precloced set in $R$ then $\{q\}$ is D-preopen set but $\{q\}$ is not D-preopen set and this contradiction. Hence $R-\{q\}$ is not D-precloced set in $R$. Since $R-\{q\} \subseteq C l_{P}^{D}(R-\{q\})$ then

$$
C l_{P}^{D}(G \cup M)=C l_{P}^{D}(R-\{q\})=R .
$$

TheOREM 4.9. For a D-metric space $(X, D)$ and $G, M \subseteq X$, the following hold:

(1) If $G \subseteq M$ then $\operatorname{Int}_{P}^{D}(G) \subseteq \operatorname{Int}_{P}^{D}(M)$.

(2) $\operatorname{Int}_{P}^{D}(G) \cup \operatorname{Int} t_{P}^{D}(M) \subseteq \operatorname{Int}_{P}^{D}(G \cup M)$.

(3) $\operatorname{Int}_{P}^{D}(G \cap M) \subseteq \operatorname{Int}_{P}^{D}(G) \cap \operatorname{Int} t_{P}^{D}(M)$.

(4) $\operatorname{Int}_{D}(G) \subseteq \operatorname{Int}_{P}^{D}(G)$.

Proof. (1) Let $x \in \operatorname{Int}_{P}^{D}(G)$. Then by Theorem 4 .6, there is D-preopen set $N$ such that $x \in N \subseteq G$ Since $G \subseteq M$ then $x \in N \subseteq M$. Hence $x \in \operatorname{Int}_{P}^{D}(\bar{M})$. That is, $\operatorname{Int}_{P}^{D}(G) \subseteq$ $\operatorname{Int}_{P}^{D}(M)$.

(2) Since $G \subseteq G \cup M$ and $M \subseteq G \cup M$, then by part(1), $\operatorname{Int}_{P}^{D}(G) \subseteq \operatorname{Int}_{P}^{D}(G \cup M)$ and $\operatorname{Int} t_{P}^{D}(M) \subseteq \operatorname{Int}_{P}^{D}(G \cup M)$. Hence $C l_{P}^{D}(G) \cup \operatorname{Int}_{P}^{D}(M) \subseteq \operatorname{Int}_{P}^{D}(G \cup \bar{M})$.

(3) Since $G \cap M \subseteq G$ and $G \cap M \subseteq M$, then by part(1), $\operatorname{Int}_{P}^{D}$ ( $G \cap$ $M) \subseteq \operatorname{Int}_{P}^{D}(G)$ and $\operatorname{Int}_{P}^{D}(G \cap M) \subseteq \operatorname{Int}_{P}^{D}(M)$. Hence $\operatorname{Int}_{P}^{D}(G \cap M) \subseteq \operatorname{Int}_{P}^{D}(G) \cap \operatorname{Int}_{P}^{D}(M)$.

(4) It is clear from Theorem 4.5 and from every D-open set is D-preopen set.

In the above theorem $\operatorname{Int}_{P}^{D}(G \cap M) \neq \operatorname{Int}_{P}^{D}(G) \cap \operatorname{Int}_{P}^{D}(M)$ as it is shown in the following example.

EXAMPLE 4.10. In Example 4.8, take $G=Q \cup\{r\}$ and $M=I R$, where $Q$ is the set of rational numbers, $I R$ is the set of irrational numbers and $r$ is any irrational number. Since $G$ and $M$ are D-preopen sets in $R$. Then $\operatorname{Int}_{P}^{D}(G) \cap \operatorname{Int}_{P}^{D}(M)=G \cap$ $M=(Q \cup\{r\}) \cap I R=\{r\}$. Since $\{r\}$ is not D-preopen set and $\operatorname{Int}_{P}^{D}(\{r\}) \subseteq\{r\}$ then $\operatorname{Int}_{P}^{D}(G \cap M)=\operatorname{Int}_{P}^{D}(\{r\})=\emptyset$.

THEOREM 4.11. For a D-metric space $(X, D)$ and $G \subseteq X$, the following hold:

(1) $\operatorname{Int}_{P}^{D}(X-G)=X-C l_{P}^{D}(G)$.

(2) $C l_{P}^{D}(X-G)=X-\operatorname{Int}_{P}^{D}(G)$.

Proof. (1) Since $G \subseteq C l_{P}^{D}(G)$, then $X-C l_{P}^{D}(G) \subseteq X-G$. Since $C l_{P}^{D}(G)$ is a D-preclosed set then $X-C l_{P}^{D}(\bar{G})$ is a Dpreopen set. Then

$$
X-C l_{P}^{D}(G)=\operatorname{Int}_{P}^{D}\left[X-C l_{P}^{D}(G)\right] \subseteq \operatorname{Int}_{P}^{D}(X-G) .
$$

For the other side, let $x \in \operatorname{Int}_{P}^{D}(X-G)$. Then there is Dpreopen set $\mathrm{N}$ such that $x \in N \subseteq X-G$. Then $X-N$ is a D-preclosed set containing $\mathrm{G}$ and $x \notin X-N$. Hence $x \notin C l_{P}^{D}(G)$, that is, $x \in X-C l_{P}^{D}(G)$.

(2) Since $\operatorname{Int}_{P}^{D}(G) \subseteq G$, then $X-G \subseteq X-\operatorname{Int}_{P}^{D}(G)$. Since $\operatorname{Int}_{P}^{D}(G)$ is a D-preopen set then $\left.X-\operatorname{Int} t_{P}^{D}(G)\right)$ is a Dpreclosed set. Then

$$
C l_{P}^{D}(X-G) \subseteq C l_{P}^{D}\left[X-\operatorname{Int}_{P}^{D}(G)\right]=X-\operatorname{Int}_{P}^{D}(G)
$$

. For the other side, let $x \notin C l_{P}^{D}(X-G)$. Then by Theorem 4.5, there is a D-preopen set $N$ containing $x$ such that $N \cap(X-G)=\emptyset$. Then $x \in N \subseteq G$, that is, $x \in \operatorname{Int}_{P}^{D}(G)$. Hence $x \notin X-\operatorname{Int}_{P}^{D}(G)$. Therefore $X-\operatorname{Int}_{P}^{D}(G) \subseteq$ $C l_{P}^{D}(X-G)$.

THEOREM 4.12. For a subset $G \subseteq X$ of D-metric space $(X, D)$ the following hold:

(1) If $M$ is a D-open set in $X$ then $C l_{P}^{D}(G) \cap M \subseteq C l_{P}^{D}(G \cap M)$. 
(2) If $M$ is a D-closed set in $X$ then $\operatorname{Int}_{P}^{D}(G \cup M) \subseteq \operatorname{Int}_{P}^{D}(G) \cup$ $M$.

Proof. (1) Let $x \in C l_{P}^{D}(G) \cap M$. Then $x \in C l_{P}^{D}(G)$ and $x \in M$. Let $V$ be any D-preopen set in $(X, D)$ containing $x$. By Theorem (3.6), $V \cap M$ is D-preopen set containing $x$. Since $x \in C l_{P}^{D}(G)$ then by Theorem 4.5$),(V \cap M) \cap G \neq \emptyset$. This implies, $V \cap(M \cap G) \neq \emptyset$. Hence by Theorem 4.5. $x \in C l_{P}^{D}(G \cap M)$. That is, $C l_{P}^{D}(G) \cap M \subseteq C l_{P}^{D}(G \cap M)$.

(2) Since $M$ is a D-closed set $X$ then by the part(1) and Theo$\operatorname{rem} 4.11$,

$$
\begin{aligned}
X-\left[\operatorname{Int}_{P}^{D}(G) \cup M\right] & =\left[X-\operatorname{Int} t_{P}^{D}(G)\right] \cap[X-M] \\
& =\left[C l_{P}^{D}(X-G)\right] \cap[X-M] \\
& \subseteq C l_{P}^{D}[(X-G) \cap(X-M)] \\
& =C l_{P}^{D}(X-(G \cup M)) \\
& =X-\left(\operatorname{Int}_{P}^{D}(G \cup M)\right) .
\end{aligned}
$$

Hence $\operatorname{Int} t_{P}^{D}(G \cup M) \subseteq \operatorname{Int}_{P}^{D}(G) \cup M$.

LEMmA 4.13. For a D-metric space $(X, D)$ and $G \subseteq X, x \in$ $C l_{D}(G)$ if and only if for all $\varepsilon>0, O_{\varepsilon}^{D}(x) \cap G \neq \emptyset$.

Proof. Let $x \in C l_{D}(G)$ and $\varepsilon>0$. If $O_{\varepsilon}^{D}(x) \cap G=\emptyset$ then $G \subseteq X-O_{\varepsilon}^{D}(x)$. Since $X-O_{\varepsilon}^{D}(x)$ is a D-closed set containing $\bar{G}$, then $C l_{D}(G) \subseteq X-O_{\varepsilon}^{D}(x)$ and $x \in C l_{D}(G) \subseteq$ $X-O_{\varepsilon}^{D}(x)$. Hence this is contradiction, because $x \in O_{\varepsilon}^{D}(x)$. Therefore $O_{\varepsilon}^{D}(x) \cap G \neq \emptyset$.

Conversely, Let $x \notin C l_{D}(G)$. Then $X-C l_{D}(G)$ is a D-open set containing $x$. Then there is $\varepsilon>0$ such that $O_{\varepsilon}^{D}(x) \subseteq X-C l_{D}(G)$ Hence by hypothesis, $O_{\varepsilon}^{D}(x) \cap G \neq \emptyset$. But this is contradiction, because $O_{\varepsilon}^{D}(x) \subseteq X-C l_{D}(G) \subseteq X-G$.

TheOrem 4.14. A subset $G \subseteq X$ of D-metric space $(X, D)$ is a D-preopen set if and only if $G \subseteq \operatorname{Int}_{D}\left(C l_{D}(G)\right)$.

Proof. Suppose that $G$ is a D-preopen set. Let $x \in G$ be arbitrary point. Then there is $\delta>0$ such that for every $y \in O_{\delta}^{D}(x)$, $O_{\varepsilon}^{D}(y) \cap G \neq \emptyset$ for every $\varepsilon>0$. By Lemma 4.13 , we get that $O_{\delta}^{D}(x) \subseteq C l_{D}(G)$. That is, $x \in \operatorname{Int}_{D}\left(C l_{D}(G)\right)$. Hence $G \subseteq \operatorname{Int}_{D}\left(C \bar{l}_{D}(G)\right)$.

Conversely, Suppose that $G \subseteq \operatorname{Int}_{D}\left(C l_{D}(G)\right)$ and $x \in G$ is arbitrary point. Then $x \in \operatorname{Int}_{D}\left(C l_{D}(G)\right)$. That is, there is $\delta>0$ such that $O_{\delta}^{D}(x) \subseteq C l_{D}(G)$. Hence for every $y \in O_{\delta}^{D}(x)$, $O_{\varepsilon}^{D}(y) \cap G \neq \emptyset$ for every $\varepsilon>0$. Hence $G$ is a D-preopen set.

For a subset $G$ of D-metric space $(X, D)$ the D-frontier operator of $\mathrm{G}$ is defined by

$$
\Gamma_{P}^{D}(G)=C l_{P}^{D}(G)-\operatorname{Int}_{P}^{D}(G) .
$$

THEOREM 4.15. For a subset $G \subseteq X$ of D-metric space $(X, D)$, the following hold:

(1) $C l_{P}^{D}(G)=\Gamma_{P}^{D}(G) \cup \operatorname{Int} t_{P}^{D}(G)$.

(2) $\Gamma_{P}^{D}(G) \cap \operatorname{Int}_{P}^{D}(G)=\emptyset$.

(3) $\Gamma_{P}^{D}(G)=C l_{P}^{D}(G) \cap C l_{P}^{D}(X-G)$.

PROOF. Note that
(1) $\Gamma_{P}^{D}(G) \cup \operatorname{Int}_{P}^{D}(G)$

$$
\begin{aligned}
& =\left(C l_{P}^{D}(G)-\operatorname{Int}_{P}^{D}(G)\right) \cup \operatorname{Int}_{P}^{D}(G) \\
& =\left[C l_{P}^{D}(G) \cap\left(X-\operatorname{Int}_{P}^{D}(G)\right)\right] \cup \operatorname{Int}_{P}^{D}(G) \\
& =\left[C l_{P}^{D}(G) \cup \operatorname{Int}_{P}^{D}(G)\right] \cap\left[\left(X-\operatorname{Int}_{P}^{D}(G)\right) \cup \operatorname{Int}_{P}^{D}(G)\right] \\
& =C l_{P}^{D}(G) \cap X=C l_{P}^{D}(G) .
\end{aligned}
$$

(2) It is clear from the definition of $\Gamma_{P}^{D}(G)$

(3) By Theorem 4.11,

$$
\begin{aligned}
\Gamma_{P}^{D}(G)= & C l_{P}^{D}(G)-\operatorname{Int}_{P}^{D}(G)=C l_{P}^{D}(G) \\
& \cap\left(X-\operatorname{Int}_{P}^{D}(G)\right) \\
= & C l_{P}^{D}(G) \cap C l_{P}^{D}(X-G) .
\end{aligned}
$$

This is the desired.

COROLlaRY 4.16. For a subset $G \subseteq X$ of D-metric space $(X, D), \Gamma_{P}^{D}(G)$ is D-preclosed set in $(X, \bar{D})$.

Proof. By Theorem 4.9 and the part(3) of the last theorem.

TheOREM 4.17. For a subset $G \subseteq X$ of D-metric space $(X, D)$, the following hold:

(1) $G$ is a D-preopen set if and only if $\Gamma_{P}^{D}(G) \cap G=\emptyset$.

(2) $G$ is a D-preclosed set if and only if $\Gamma_{P}^{D}(G) \subseteq G$.

(3) $G$ is both D-preopen set and D-preclosed set if and only if $\Gamma_{P}^{D}(G)=\emptyset$.

Proof. (1) Let $G$ be a D-preopen set. Then $\operatorname{Int} t_{P}^{D}(G)=G$. Then by Theorem 4.15,

$$
\Gamma_{P}^{D}(G) \cap G=\Gamma_{P}^{D}(G) \cap \operatorname{Int}_{P}^{D}(G)=\emptyset
$$

Conversely, suppose that $\Gamma_{P}^{D}(G) \cap G=\emptyset$. Then

$$
\begin{aligned}
G-\operatorname{Int}_{P}^{D}(G) & =\left[G \cap C l_{P}^{D}(G)\right]-\left[G \cap \operatorname{Int}_{P}^{D}(G)\right] \\
& =G \cap\left(C l_{P}^{D}(G)-\operatorname{Int}_{P}^{D}(G)\right) \\
& =G \cap \Gamma_{P}^{D}(G)=\emptyset .
\end{aligned}
$$

That is, $\operatorname{Int} t_{P}^{D}(G)=G$. Hence $G$ is a D-preopen set.

(2) Let $G$ be a D-preclosed set. Then $C l_{P}^{D}(G)=G$. Then

$$
\Gamma_{P}^{D}(G)=C l_{P}^{D}(G)-\operatorname{Int}_{P}^{D}(G)=G-\operatorname{Int} t_{P}^{D}(G) \subseteq G .
$$

Conversely, let $\Gamma_{P}^{D}(G) \subseteq G$. Then by Theorem 4.15,

$$
C l_{P}^{D}(G)=\operatorname{Int}_{P}^{D}(G) \cup \Gamma_{P}^{D}(G) \subseteq \operatorname{Int}_{P}^{D}(G) \cup G \subseteq G .
$$

That is, $C l_{P}^{D}(G)=G$. Hence $G$ is D-preclosed set.

(3) Let $G$ be both D-preclosed set and D-preopen set. Then $C l_{P}^{D}(G)=G=\operatorname{Int}_{P}^{D}(G)$. Then

$$
\Gamma_{P}^{D}(G)=C l_{P}^{D}(G)-\operatorname{Int}_{P}^{D}(G)=G-G=\emptyset .
$$

Conversely, suppose that $\Gamma_{P}^{D}(G)=\emptyset$. Then $C l_{P}^{D}(G)-$ $\operatorname{Int} t_{P}^{D}(G)=\emptyset$. Since $\operatorname{Int}_{P}^{D}(G) \subseteq C l_{P}^{D}(G)$ then $C l_{P}^{D}(G)=$ $\operatorname{Int}_{P}^{D}(G)$. Since $\operatorname{Int}_{P}^{D}(G) \subseteq G \subseteq C l_{P}^{D}(G)$ then

$$
C l_{P}^{D}(G)=G=\operatorname{Int}_{P}^{D}(G) .
$$

That is, $C l_{P}^{D}(G)=G$. Hence $G$ is both D-preclosed set and Dpreopen set. 


\section{REFERENCES}

[1] A. A. Ali Fora, M. O. Massadeh and M. S. Bataineh, A new Structure and Contribution in D-metric Spaces, British Journal of Mathematics and Computer Science, 22(1), (2017), 1-9.

[2] B. C. Dhage, Generalized Metric Space and Topological Structure I, Analele Stiitifice Ale Univ. Cuza Iasi Mat., 46, (2000), 3-24.

[3] B. C. Dhage, (1984), A study of some fixed point theorms, Ph.D.Thesis. Marathwada Univ. Aurangabad, India.

[4] C. D. Bele and U. P. Dolhare, An Extension of Common Fixed Point Theorem in D-Metric Space, International Journal of Mathematics and its Applications, 5, (2017), 13-18.

[5] C. G. Aras, S. Bayramov and M. I. Yazar, Soft D-Metric Spaces, Bol. Soc. Paran. Mat, 38, (2020), 137-147.

[6] I. Ramabhadrasarma and S. Sambasivarao, On D-metric spaces, Journal of Global Research in Mathematical Archives, 12, (2013), 31-39.

[7] J. Muscat, (2016), Function anaylsise, Springer Heidelberg New York, London. 